\title{
Osteoporose em mulheres na pós-menopausa, cálcio dietético e outros fatores de risco
}

\section{Osteoporosis in postmenopausal women, dietary calcium and other risk factors}

\author{
Haydée Serrão LANZILLOTTI ${ }^{1}$ \\ Regina Serrão LANZILLOTT| ${ }^{2}$ \\ Ana Paula Rocha TROTTE ${ }^{3}$ \\ Alessandra Silva DIAS 3 \\ Bruna BORNAND 4 \\ Eduardo André Moura Martins COSTA ${ }^{4}$
}

\section{R E S U M O}

O objetivo deste trabalho foi examinar os principais fatores de risco associados ao desenvolvimento de osteoporose primária em mulheres na pós-menopausa com osteopenia. Setenta e seis pacientes entre 46 e 85 anos foram selecionadas; $56,6 \%$ apresentaram diagnóstico de osteopenia e 43,4\%, de osteoporose, de acordo com o critério da Organização Mundial da Saúde. Os fatores de risco foram pesquisados por meio de registro clínico e questionário de freqüência alimentar. 0 odds ratio foi calculado por meio do aplicativo Statistica. Oitenta e seis por cento das mulheres com osteopenia e $84,8 \%$ das com osteoporose apresentaram baixa ingestão de cálcio através de produtos lácteos. 0 teste " $\mathrm{t}$ " para amostras independentes foi aplicado e não inferiu diferença significativa $(p=0,99)$ entre os dois grupos. No grupo com osteopenia, os fatores de risco assumiram a seguinte forma hierárquica: ausência de terapia de reposição hormonal $(2,000)$, não-exposição ao sol $(1,516)$, consumo de bebidas alcoólicas na juventude $(1,346)$, consumo atual inadequado de cálcio $(1,163)$, ausência de atividade física atual $(1,145)$, história familiar de osteoporose $(1,101)$, ausência de atividade física na juventude $(1,006)$, tabagismo $(0,851)$ e consumo atual de bebidas alcoólicas $(0,827)$. Em conclusão, a ausência de terapia de reposição hormonal foi o fator de risco que indicou maior probabilidade de ocorrência de osteoporose entre as mulheres com osteopenia.

Termos de indexação: osteoporose, cálcio dietético, fatores de risco, osteoporose pós-menopausa.

\footnotetext{
1 Departamento de Nutrição, Universidade Gama Filho. Rua São Francisco Xavier, n. 524, 21, Maracanã, Rio de Janeiro, RJ, Brasil; Instituto de Nutrição, Universidade do Estado do Rio de Janeiro. Correspondência para/Correspondence to: H.S. LANZILLOTTI. E-mail: haydeelan@uol.com.br

2 Instituto de Matemática e Estatística, Universidade do Estado do Rio de Janeiro.

3 Instituto de Nutrição, Universidade do Estado do Rio de Janeiro. Bolsista de Iniciação Científica.

${ }^{4}$ Instituto de Matemática e Estatística, Universidade do Estado do Rio de Janeiro. Estagiário de pesquisa SR2/UERJ.
} 


\section{A B S T R A C T}

The objective of this paper was to examine the main risk factors associated with the development of primary osteoporosis in postmenopausal women with osteopenia. Seventy-six patients between 46 and 85 years old were selected; $56.5 \%$ presented an osteopenia diagnosis and $43.4 \%$ presented an osteoporosis diagnosis, according to the World Health Organization criterion. The risk factors were investigated through clinical register and food-frequency questionnaire. The odds ratio was calculated by the software Statistica. Eighty-six percent of the women affected by osteopenia and $84.8 \%$ of those suffering from osteoporosis showed low intake of calcium through dairy products. The " $t$ " test for independent samples was applied and no significant difference ( $p=0.99$ ) between the two groups was found. In the group with osteopenia, the risk factors took the following hierarchical form: absence of hormonal replacement therapy (2.000), no sun exposure (1.516), alcoholic drink consumption in youth (1.346), current inadequate intake of calcium (1.163), absence of physical activities in the present (1.145), family history of osteoporosis (1.101), absence of physical activities in youth (1.006), smoking habit $(0.851)$ and consumption of alcoholic drink in the present (0.827). It was concluded that the absence of hormonal replacement therapy was the risk factor which indicated the major probability of the osteoporosis occurrence among the women with osteopenia.

Index terms: osteoporosis, calcium, dietary, risk factors, osteoporosis, postmenopausal.

\section{N T R O D U ÇÃ O}

A osteoporose é uma doença esquelética sistêmica, caracterizada por massa óssea baixa e deterioração microarquitetural do tecido ósseo, conduzindo à fragilidade do osso e ao aumento do risco de fratura (Shaw $\&$ Witzke, 1998).

Segundo dados epidemiológicos para os Estados membros da União Européia, haverá mudanças na pirâmide etária, com concentração mais acentuada no grupo de 80 anos e mais. Neste grupo haverá maior incidência de fraturas osteoporóticas. Esta faixa populacional passará de 8,9 milhões de mulheres e 4,5 milhões de homens em 1995 a 26,4 milhões de mulheres e 17,4 milhões de homens em 2050. Estima-se que as fraturas vertebrais passarão de 23,7 milhões no ano de 2000 para 37,3 milhões em 2050, representando um aumento de $50 \%$. O comportamento das fraturas de quadris, associadas à osteoporose, passará de 414,1 milhões para 972 milhões, representando um aumento de 134,0\% (Comisión Europea, 1998). Nos Estados Unidos da América a incidência das fraturas osteoporóticas oscilam ao redor de 700 mil fraturas/ano (Litvak, 1990).
Existem grandes lacunas nos dados epidemiológicos internacionais sobre a prevalência de osteoporose e identificação prospectiva sobre os riscos de fratura. No entanto, mesmo sem um sistema de vigilância efetivo, pode-se inferir que a modificação da pirâmide etária conferirá importância ainda maior à osteoporose em um futuro próximo. Indiscutivelmente, no desfecho da osteoporose, a idade é de longe o mais importante determinante de massa óssea. A diminuição da densidade óssea com a idade reflete o efeito somatório de vários processos, os quais ocorrem universalmente, porém, em proporções diferentes de acordo com variações individuais (Marques Neto \& Lederman, 1995).

Na menopausa aumenta a renovação e diminui a formação óssea em cada unidade de remodelação, o que conduz a uma perda de massa óssea. 0 risco de osteoporose depende tanto da massa óssea máxima alcançada nos anos da idade adulta jovem quanto do índice de perda da massa nas épocas posteriores. 0 pico de massa óssea geralmente não é alcançado antes de 30 anos e o estilo de vida é um importante determinante da probabilidade de desenvolver mais tarde osteoporose. Dentre os fatores de risco 
está a ausência de atividade física regular e de terapia de reposição hormonal, bem como fatores genéticos e os relativos à dieta (Hallberg et al.,1992). Não se sabe com certeza em que idade começa a perda óssea, mas acredita-se que, entre 40 anos e a menopausa, as mulheres perdem aproximadamente $0,3 \%$ a $0,5 \%$ de sua massa de osso cortical por ano; após a menopausa, este ritmo acelera para 2\% a 3\% ao ano (Lucasin Junior \& Lima, 1994).

O exercício transmite carga ao esqueleto mediante o impacto direto e a contração muscular. A falta de atividade física adequada pode influenciar negativamente o pico de massa óssea, havendo a necessidade de incentivo à prática esportiva para mulheres em todas as idades (Prince et al., 1995). A sustentação para a afirmativa de que o exercício físico é uma medida preventiva para a osteoporose baseia-se em observações segundo as quais indivíduos fisicamente ativos e tipicamente atletas têm maior massa óssea em relação aos sedentários (Drinkwater et al., 1995). Exercícios de força e de carga de alto impacto garantem melhores resultados, em comparação com os de treinamento de resistência (Shaw \& Witzke, 1998). Se o exercício está vinculado ao suplemento de cálcio, atingindo um consumo diário de aproximadamente $1800 \mathrm{mg} / \mathrm{dia}$, reduzse significativamente a perda óssea no sítio dos quadris (Prince et al., 1995). No entanto, não existe nenhuma evidência de que o exercício sozinho possa reduzir a perda óssea associada à redução dos hormônios reprodutivos, ocorrência característica da menopausa.

$\mathrm{Na}$ menopausa, a principal alteração biológica é o cessar da ovulação, confirmada quando a menstruação se interrompe por pelo menos um ano. Após a menopausa, os ovários tornam-se inativos e ocorre mínima ou nenhuma liberação de estrogênio, coincidindo com a redução da absorção de cálcio pelo intestino, devido à baixa produção de calcitonina, hormônio que inibe a desmineralização óssea (Ulrich et al., 1996; Nieves et al., 1998). 0 déficit de estrogênio é um determinante importante na perda óssea durante a menopausa e, quando precoce, o risco é muito maior (Shaw $\&$ W itzke, 1998). De acordo com estudo de meta-análise realizado por Nieves et al. (1998), o efeito da associação do estrogênio com um consumo de cálcio adequado (aproximadamente $1200 \mathrm{mg} /$ dia) parece mais benéfico do que a soma de cada efeito sozinho, induzindo haver interação entre os fatores de risco.

Apesar de não se compreender plenamente o mecanismo de regulação da massa óssea máxima, sabe-se que o fator genético é um agente importante na predisposição à osteoporose. Khoury (1998), através de meta-análise, encontrou evidências fortes sugerindo que a interação entre genes e ambiente desempenha um papel na densidade mineral óssea. Em dezembro de 1997 foram listados no Online Mendelian Inheritance in Men (OMIM) 67 genes associados com a osteoporose, incluindo a Vitamin D receptor gene (VDR). No entanto, não está claro como eles interagem com o cálcio (Khoury 1998). Revisão realizada por Zmuda et al. (2000) aponta a relação entre o fator genético e a osteoporose. Assim, a Vitamina $D$ regula a homeostase do cálcio e a mineralização óssea e sua ação é mediada através do VDR gene localizado no cromossoma $12 q$, o qual tem variantes alélicas conhecidas. O VDR pertence à família de receptores de hormônios tireóides e esteróides e medeia os efeitos da 1,25 dihydroxy vitamin $D\left[1,25(\mathrm{OH})_{2} \mathrm{D}\right]$ na expressão gênica. Os autores ainda revelaram que há indícios de associação entre a perda óssea em mulheres na pós-meno-pausa e o VDR gene. 0 componente hereditário é um fator difícil de ser analisado, mas a história familiar de osteoporose pode ser considerada um indicador adequado para sua avaliação.

Há grande variação individual na absorção de cálcio, a qual está condicionada à presença de Vitamina $D$ e ao aporte de cálcio na alimentação. As taxas de absorção de cálcio pelo intestino oscilam entre $30 \%$ e $50 \%$ (Amaya-Farfan, 1994). É necessário haver um suprimento constante de cálcio dietético biodisponível para garantir a massa óssea máxima do programa 
genético individual. A importância da exposição ao sol está relacionda à Vitamina $D$, que é obtida com a alimentação através da síntese cutânea na presença da radiação ultravioleta da luz solar. A Vitamina $D$ se converte em 25 -hidroxyvitamina D (250HD, calcidiol) no fígado; os níveis de calcidiol no sangue constituem uma valoração razoavelmente exata do nível de vitamina $D$. A forma biologicamente ativa da Vitamina $D, a$ 1,25-dihydroxyvitamina $\mathrm{D}_{3}\left(1,25(\mathrm{OH})_{2} \mathrm{D}_{3}\right.$, calcitriol), se forma no rim a partir do calcidiol, 0 qual estimula a reabsorção óssea e a absorção intestinal do cálcio, conduzindo a um incremento da concentração do cálcio sérico. No entanto, 0 aumento da concentração de cálcio sérico freia a produção do hormônio paratireóide e a síntese de calcitriol, produzindo uma maior excreção urinária de cálcio e a redução tanto da reabsorção óssea quanto da absorção intestinal de cálcio (Comisión Europea, 1998). A deficiência de vitamina $D$ nas pessoas de idade avançada pode não ser puramente decorrente de um aporte insuficiente, mas da diminuição da síntese renal de 1,25-dihydroxyvitamina $D$, apontada como causadora de menor proteína ligante de cálcio na estrutura óssea, provocando excessiva perda urinária de cálcio (Kung et al., 1998).

Tem-se demonstrado que uma alta ingestão de fosfato através dos alimentos conduz a um aumento da excreção do hormônio paratireóide, apresentando um efeito inibidor sobre a formação óssea (Coelho, 1995; Kärlkäinen \& Lamberg-Allardt, 1996).

Os lipídeos dietéticos, particularmente triglicerídeos e ácidos graxos saturados de baixo peso molecular, formam sabões insolúveis com o cálcio dietético, aumentando sua excreção fecal e conseqüentemente reduzindo sua absorção (Coelho, 1995).

0 ácido oxálico, presente em alguns vegetais (beterraba, espinafre, semente de tomate, aspargo) e também no cacau, chocolate, gérmen de trigo, nozes e feijões, forma complexos com o cálcio dietético, os quais precipitam no lúmen intestinal em conseqüência do meio alcalino e são excretados pelas fezes (Coelho, 1995).

Os fitatos representam compostos formados durante o processo de maturação de sementes e grãos de cereais integrais e feijões, podendo se complexar com minerais como o cálcio, o ferro, o zinco e com as proteínas. Em virtude da presença de fitato, o balanço de cálcio tem sido alterado por dietas ricas em fibras (Haack et al., 1998).

Quanto à cafeína, encontrada nas bebidas como café, chás, chocolate e bebidas gasosas cafeinadas, são contraditórios os dados epidemiológicos sobre a relação entre seu consumo e a formação de massa óssea. Segundo Harris \& Dawson-Hughes (1994), uma ingestão ótima de cálcio por mulheres na pós-menopausa poderia proteger contra os efeitos danosos da cafeína no osso. Por outro lado, de acordo com Weaver et al. (1999), um copo de $240 \mathrm{~mL}$ de café reduz, em média, a retenção de cálcio em 2-3mg, o que não conduziria ao comprometimento da massa óssea.

Embora a causa da remodelação anormal do osso não seja bem entendida, uma feição comum do processo osteoporótico é o balanço de cálcio negativo, devido à maior perda do que retorno deste mineral no esqueleto (Kung et al., 1998). A ingestão de cálcio a partir do nascimento, principalmente na infância e juventude, aliada ao exercício físico regular, é um fator de prevenção de uma posterior desmineralização óssea (Ulrich, 1996).

Entre os fatores de risco modificáveis estão também incluídos o tabagismo e o álcool (Khoury, 1998). O efeito do álcool sobre a função osteoplástica está vinculado às disfunções hepáticas, como esteatose hepática, hepatite alcoólica e cirrose, ocasionadas pelo consumo exagerado, enquanto a ação do tabagismo está relacionada ao efeito inibidor direto do tabaco nos osteoblastos e à menopausa mais precoce entre as mulheres fumantes (Comisión Europea, 1998). 
Sendo a osteoporose na pós-menopausa uma enfermidade multicausal, este estudo objetivou examinar os principais fatores de risco para o desenvolvimento da osteoporose primária em mulheres na pós-menopausa, em um grupo de pacientes com osteopenia.

\section{CASUÍSTICA E MÉTODOS}

Foi conduzido um estudo transversal retrospectivo, reunindo na amostra acidental 76 pacientes do sexo feminino, entre 46 e 85 anos, clientes de uma clínica de diagnóstico situada em um bairro de classe média e média alta da cidade do Rio de Janeiro. Foram excluídas da amostra mulheres com osteoporose secundária, ou seja, aquelas que utilizavam cortisona, apresentavam quadro de hipertireoidismo, artrite reumatóide, espondilite anquilosante e doença de Paget. As participantes foram informadas acerca dos objetivos e procedimentos do estudo para a obtenção do "consentimento esclarecido".

Os diagnósticos de osteopenia e osteoporose foram realizados através de densiometria de dupla energia de Raios X (DEXA), permitindo medir a densidade mineral óssea Bone Mineral Density (BMD) com o uso do aparelho Lumar CO.DPXL. Os critérios densiométricos adotados para os diagnósticos foram os sugeridos por Kanis et al. (1994) e adotados pela Organização M undial da Saúde (OM S) (Comisión Europea, 1998). Estes critérios são baseados em pontos de desvio-padrão, expressos em relação aos dados de referência de mulheres na pré-menopausa sãs. M ediante o uso de unidades de desvio-padrão, evitam-se os problemas relacionados com as diferenças de calibre dos instrumentos. A classificação estabelece as seguintes categorias:

A) Normal: valor de BMD maior do que -1DP inclusive;

B) Osteopenia (massa óssea reduzida): valor de BMD entre -2,5DP inclusive e -1DP exclusive;
C) Osteoporose: valor de BMD menor que -2,5DP exclusive;

D) Osteoporose severa (osteoporose estabelecida): valor de BMD menor que -2,5 DP, com presença de uma ou mais fraturas.

A determinação do consumo de cálcio da dieta e de seus modificadores de absorção foi possível através do questionário de freqüência alimentar validado por Heath et al. (2000), os quais, a partir de metodologia sugerida por Willet (1990), construíram um questionário direcionado à estimativa de ferro da dieta e de seus modificadores da absorção. A maioria desses instrumentos estima o consumo de todos os grupos de alimentos, mas nenhum deles tentou estimar os nutrientes separadamente. Esta informação é necessária, se é desejável identificar a possibilidade de biodisponibilidade de um nutriente. Heath et al. (2000) utilizaram o questionário de freqüência alimentar de ferro para investigar a etiologia e o tratamento de mulheres adultas pré-anêmicas. Este estudo adaptou-o para mulheres na pós-menopausa sob o risco de osteoporose. Willet (1990) sugere questionário de freqüência alimentar para estabelecer o perfil de freqüência no período de um ano. Analogamente a Heath et al. (2000), escolheu-se o período de um mês, pois é improvável que indivíduos tenham memória acurada para recordar o consumo de alimentos por mais de um mês, especialmente em mulheres na faixa etária objeto do estudo, cuja memória recente é precária (Haines et al., 1994).

Os alimentos lácteos (leite integral, leite desnatado, iogurte, coalhada, queijo minas, queijo prato, requeijão) consumidos no desjejum, almoço, lanche, jantar e ceia foram quantificados em medidas caseiras e estas transformadas em gramas com o auxílio da Tabela para Avaliação de Consumo Alimentar em Medidas Caseiras (Pinheiro et al.,1994). As entrevistadas responderam sobre a freqüência de consumo: diária, de 3 a 4 vezes por semana, 1 a 2 vezes por semana, 1 vez por mês e não usa. 0 cálculo para avaliar o teor de cálcio da dieta valeu-se das 
Tabelas de Composição de Alimentos do Instituto Brasileiro de Geografia e Estatística (Instituto Brasileiro...,1996) e da Tabela de Composição Química dos Alimentos (Franco, 1998).

A absorção de nutrientes é influenciada por uma série de fatores e a composição de cada refeição é o principal determinante das interações intraluminares e de sua influência negativa ou positiva sobre o processo de utilização dos nutrientes nela contidos (Coelho, 1995). A partir desta premissa, o questionário de freqüência alimentar da ingestão de alimentos lácteos foi estruturado para ser levantado por refeição. Os fatores anti-nutricionais, presentes nas refeições, foram identificados de forma dicotômica. Os questionários foram aplicados por acadêmicos de Nutrição devidamente treinados.

As informações sobre os fatores de riscos, além da dieta, foram também levantadas pelos acadêmicos de Nutrição na Ficha Cadastral da Clínica e através do preenchimento do Formulário Complementar de Entrevista.

Foram obtidos dados sobre tabagismo, ausência de atividade física na juventude e atualmente, consumo de álcool na juventude e atualmente, história familiar de osteoporose, não adoção de terapia de reposição hormonal e nãoexposição ao sol.

Considerou-se como tabagismo o uso de mais de 20 cigarros por dia (Instituto Nacional..., 2001) e etilismo, o consumo superior a um cálice de vinho ou cerveja nas grandes refeições e/ou uma dose de bebida destilada três vezes por semana por mais de dois anos. Os entrevistadores procuravam saber se na infância e atualmente os participantes faziam exercícios regulares, ou seja, pelo menos três dias por semana (Shaw $\&$ Witzke, 1998), preferencialmente os que exigem suportar carga, como ginástica ou jardinagem (Drinkwater et al., 1995), devido ao estresse mecânico ao qual o osso é submetido (Lucasin \& Lima, 1994). Também foi perguntado sobre caminhada, corrida e jogos, porque estas são atividades habituais entre pessoas idosas. Sobre a terapia de reposição hormonal, os pesquisadores verificaram na Ficha
Cadastral da Clínica se as participantes faziam uso de hormônios sexuais após a menopausa. A terapia de reposição hormonal deve ser prolongada tanto quanto possível. 0 período de cinco a dez anos, com acompanhamento médico regular, é normalmente desejável. Nieves et al. (1998) indicam haver uma relação sinergética entre um alto consumo de cálcio e a terapia com hormônios sexuais, propiciando efeitos maiores sobre a redução da perda óssea no tratamento da osteoporose.

A história familiar de osteoporose foi levantada durante a entrevista com as senhoras, quando se averiguava se parentes próximos (mãe, pai, avô, avó, filho, filha, irmão, irmã) já haviam sido diagnosticados com osteopenia ou osteoporose. A não-exposição ao sol foi considerada quando a participante raramente saía de casa durante o dia para especificamente tomar sol ou fazer outra atividade sob a luz natural.

\section{RESULTA DOSE DISCUSSÃ O}

Das 76 mulheres participantes desta pesquisa, 56,6\% apresentaram osteopenia e $43,4 \%$, osteoporose. Estudo realizado por Borges et al. (2000), em Santa Catarina, com 60 mulheres na pós-menopausa, na faixa etária entre 50 e 65 anos, identificou $23,3 \%$ como sem osteopenia e sem osteoporose, 58,3\% com osteopenia e 18,4\% com osteoporose.

Há controvérsia quanto à recomendação da ingestão de cálcio para mulheres na pós-menopausa. Embora a Recommended Dietary Allowances (RDA) de 1989 (Lucas, 1998) indique uma ingestão de $1200 \mathrm{mg}$ para mulheres a partir de 50 anos, Dawson-Hughes (1998) e Whiting (1999) sugerem $1000 \mathrm{mg}$ de cálcio por dia para aquelas recebendo reposição hormonal, mas na ausência desta terapia, eles recomendam 1500mg de cálcio/dia, enquanto Prince et al. (1995) indicam cotas de aproximadamente $1800 \mathrm{mg}$. Mulheres chinesas na pós-menopausa com osteoporose responderam com concentração de 
cálcio sérico significativamente reduzida, quando recebiam 1200mg de cálcio (Kung et al., 1998). Neste estudo, o ponto de corte adotado para o valor requerido foi entre $1000 \mathrm{mg}$ e $1500 \mathrm{mg} / \mathrm{dia}$ (Whiting, 1999).

Tanto as mulheres com osteopenia quanto as com osteoporose apresentaram consumo de cálcio abaixo do recomendado (Tabelas 1 e 2). Os altos coeficientes de variação indicam grande variabilidade de ingestão deste mineral entre elas. 0 teste " $\mathrm{t}$ " de Student para amostras independentes revelou não haver diferença significativa de consumo entre os grupos.

No estudo já citado, Borges et al. (2000) identificaram entre as mulheres sem osteopenia e sem osteoporose $(85,7 \%$ ) um consumo de cálcio significativamente maior do que nas mulheres com diagnóstico; enquanto aquelas preencheram $148,0 \%$ das necessidades diárias de cálcio, as com osteopenia e com osteoporose, atingiram apenas $87,0 \%$ e $58,0 \%$, respectivamente. Kung et al. (1998) indicaram consumo de $561 \pm 183 \mathrm{mg} / \mathrm{d}$ entre 25 mulheres chinesas com osteoporose na pós-menopausa e ainda uma excepcional absorção potente de cálcio. Outra pesquisa, realizada por Haines et al. (1994), também com mulheres chinesas na pós-menopausa, revelou consumos médios ainda mais baixos. Quando aferido por recordatório de 24 horas, os valores alcançaram 397,6mg, e pelo questionário de freqüência alimentar chegaram a 390,9mg.

Menos de $10 \%$ das mulheres, em ambos os grupos avaliados neste trabalho, ingerem com a freqüência menor que três vezes por semana produtos lácteos (iogurte, queijo minas, queijo prato) no almoço e/ou jantar (Tabela 3). Este achado permite inferir sobre a não-competitividade do ferro em relação ao cálcio consumido (Hallberg et al., 1992; Whitting, 1999).

Algumas entrevistadas consomem produtos lácteos nas grandes refeições, permitindo advertir que o hábito de comer sobremesas como iogurte, doce com queijo e doces feitos com leite deve ser evitado, dando-se preferência ao seu consumo duas horas após as grandes refeições (Coelho, 1995) (Tabela 3). Ainda se pode verificar que, entre as mulheres com osteopenia, a maioria faz uso expressivo de queijo minas, havendo equilíbrio no uso de leite desnatado e integral. Entretanto, no grupo com osteoporose, o consumo se distribui entre o leite desnatado e o queijo minas, sendo o leite integral bem menos consumido. A opção pelo leite desnatado favorece a absorção do cálcio, em virtude da não-formação de sabões insolúveis com o cálcio dietético (Coelho, 1995).

Tabela 1. Consumo diário de cálcio* em mulheres na pós-menopausa.

\begin{tabular}{lcc}
\hline Estatísticas $(\mathrm{mg})$ & Osteopenia $(\mathrm{n}=43)$ & Osteoporose $(\mathrm{n}=33)$ \\
\hline Média & 630,15 & 630,15 \\
Mediana & 590,40 & 590,40 \\
Desvio-padrão & 555,36 & 468,13 \\
Coeficiente de variação & 88,13 & 74,29 \\
\hline
\end{tabular}

${ }^{*}$ Freqüência de consumo em pelo menos 3 vezes por semana.

Tabela 2. Classificação das mulheres pós-menopausa segundo o consumo de cálcio* em relação à recomendação** para este micronutriente.

\begin{tabular}{|c|c|c|c|c|c|c|}
\hline \multirow{2}{*}{ Enfermidade } & \multicolumn{2}{|c|}{ Abaixo do recomendado } & \multicolumn{2}{|c|}{ Recomendado } & \multicolumn{2}{|c|}{ Acima do recomendado } \\
\hline & $n$ & $\%$ & $\mathrm{n}$ & $\%$ & $n$ & $\%$ \\
\hline Osteopenia & 37 & 86,0 & 4 & 9,3 & 2 & 4,7 \\
\hline Osteoporose & 28 & 84,8 & 3 & 9,1 & 2 & 6,1 \\
\hline
\end{tabular}

(*) Freqüência de consumo em pelo menos 3 vezes por semana; (**) Recomendação de ingestão diária de cálcio para mulheres pós-menopausa:1000-1500mg (Dawson-Hughes,1998; Whiting, 1999). 
Tabela 3. Freqüência de consumo de alimentos ricos em cálcio* em mulheres na pós-menopausa.

\begin{tabular}{|c|c|c|c|c|c|c|c|c|c|c|c|c|}
\hline \multirow{2}{*}{ Osteopenia $(n=43)$} & \multicolumn{2}{|c|}{ Desjejum } & \multicolumn{2}{|c|}{ Almoço } & \multicolumn{2}{|c|}{ Lanche } & \multicolumn{2}{|c|}{ Jantar } & \multicolumn{2}{|c|}{ Ceia } & \multicolumn{2}{|c|}{ Não usa } \\
\hline & $n$ & $\%$ & $n$ & $\%$ & $\mathrm{n}$ & $\%$ & $n$ & $\%$ & $n$ & $\%$ & $n$ & $\%$ \\
\hline \multicolumn{13}{|l|}{ Alimentos } \\
\hline Leite integral & 13 & 30,2 & - & - & 4 & 9,3 & 1 & 2,3 & 1 & 2,3 & 29 & 67,4 \\
\hline Leite desnatado & 22 & 51,2 & - & - & 7 & 16,3 & - & - & 10 & 23,3 & 18 & 41,9 \\
\hline logurte & 7 & 16,3 & 2 & 4,7 & 7 & 16,3 & 1 & 2,3 & 2 & 4,7 & 26 & 60,5 \\
\hline Coalhada & 2 & 4,7 & - & - & 2 & 4,7 & - & - & - & - & 37 & 86,0 \\
\hline Queijo Minas & 35 & 81,4 & 1 & 2,3 & 29 & 67,4 & 4 & 9,3 & 1 & 2,3 & 3 & 7,0 \\
\hline Queijo Prato & 9 & 20,9 & 4 & 9,3 & 10 & 23,3 & 1 & 2,3 & - & - & 26 & 60,5 \\
\hline Requeijão & 17 & 39,5 & - & - & 13 & 30,2 & 1 & 2,3 & 2 & 4,7 & 18 & 41,9 \\
\hline Ricota & 2 & 4,7 & - & - & - & - & - & - & - & - & 40 & 93,0 \\
\hline \multirow{2}{*}{ Osteoporose ( $n=33)$} & \multicolumn{2}{|c|}{ Desjejum } & \multicolumn{2}{|c|}{ Almoço } & \multicolumn{2}{|c|}{ Lanche } & \multicolumn{2}{|c|}{ Jantar } & \multicolumn{2}{|c|}{ Ceia } & \multicolumn{2}{|c|}{ Não usa } \\
\hline & $\mathrm{n}$ & $\%$ & $n$ & $\%$ & $\mathrm{n}$ & $\%$ & $\mathrm{n}$ & $\%$ & $\mathrm{n}$ & $\%$ & $\mathrm{n}$ & $\%$ \\
\hline \multicolumn{13}{|l|}{ Alimentos } \\
\hline Leite integral & 9 & 27,3 & - & - & 3 & 9,1 & - & - & 4 & 12,1 & 22 & 66,7 \\
\hline Leite desnatado & 18 & 54,5 & - & - & 6 & 18,2 & - & - & 5 & 15,2 & 13 & 39,4 \\
\hline logurte & 9 & 27,3 & 1 & 3,0 & 8 & 24,2 & 1 & 3,0 & 1 & 3,0 & 16 & 48,5 \\
\hline Coalhada & 4 & 12,1 & - & - & 3 & 9,1 & - & - & - & - & 27 & 81,8 \\
\hline Queijo Minas & 22 & 66,7 & 2 & 6,1 & 13 & 39,4 & - & - & 2 & 6,1 & 4 & 12,1 \\
\hline Queijo Prato & 10 & 30,3 & 1 & 3,0 & 9 & 27,3 & - & - & 1 & 3,0 & 18 & 54,5 \\
\hline Requeijão & 9 & 27,3 & - & - & 9 & 27,3 & 1 & 3,0 & - & - & 20 & 60,6 \\
\hline Ricota & 1 & 3,0 & - & - & 1 & 3,0 & - & - & - & - & 22 & 66,7 \\
\hline
\end{tabular}

${ }^{(*)}$ Freqüência de consumo em pelo menos 3 vezes por semana.

A indicação de ingestão de produtos lácteos para a população brasileira, encontrada nos guias alimentares (Philippi et al., 1999), é de três porções diárias. Um copo médio de leite $(200 \mathrm{~mL})$ contém 228mg de cálcio; 15 gramas de queijo, 95,25mg; $120 \mathrm{~mL}$ de iogurte, $144 \mathrm{mg}$ (Franco, 1998). Assim, para atingir os $1000 \mathrm{mg}$ a $1500 \mathrm{mg}$ seria necessária a ingestão de seis a dez porções diárias de alimentos lácteos, as quais somariam aproximadamente 42 a 70 gramas de proteínas. Deve-se tomar cuidado com as quantidades ingeridas de produtos lácteos, pois a elevação indiscriminada do número de porções poderia levar à ingestão protéica excessiva. Dietas hiperprotéicas podem aumentar a perda de cálcio urinário e, portanto, seriam perigosas por causa do potencial para acelerar a progressão da osteoporose (Lemon, 1998). Desse modo, o estabelecimento de um balanço negativo de cálcio em indivíduos que consomem dietas hiperprotéicas por longos períodos, poderia ser apontado como um dos fatores de risco da osteoporose (Coelho, 1995; Khoury, 1998). Estes achados mostram a dificuldade de alcançar as recomendações de cálcio sugeridas unicamente com a dieta. No entanto, Prince et al. (1995) conseguiram resultados positivos através do suplemento de dietas com leite em pó para prevenir a perda óssea pós e pré-menopausa.

O consumo de pão integral atingiu, em ambos os grupos, valores superiores a $30,0 \%$ (Tabela 4). No pão, a presença de fitato está atenuada pelo processo de fermentação, conforme relatado na revisão realizada por Silva $\&$ Silva (1999). 0 consumo de biscoitos de fibras $(11,6 \%$ e $9,1 \%$ das mulheres com osteopenia e osteoporose, respectivamente) e arroz integral $(4,7 \%$ e $9,1 \%$ das mulheres com osteopenia e osteoporose, respectivamente) é discreto entre as entrevistadas. A venell et al. (1994) demonstraram que o uso de dietas ricas em fibras na redução de peso em mulheres na pós-menopausa aumenta 
significativamente a perda óssea, conduzindo ao risco de desenvolvimento de osteoporose. Entretanto, para Haack et al. (1998) a mistura de fibras alimentares na dieta tem pouco efeito sobre o balanço de cálcio, quando o consumo é igual ou superior a $1500 \mathrm{mg} / \mathrm{dia}$. Ressalta-se que no presente estudo as médias de consumo de cálcio foram de $630,15 \mathrm{mg}$ e $630,10 \mathrm{mg}$ para os grupos com osteopenia e osteoporose, respectivamente. Se este perfil de consumo de cálcio for acompanhado por alta ingestão de fibras, a dieta torna-se um fator de risco.

Dentre os alimentos ricos em ácido oxálico, o tomate liderou o hábito de consumo para ambos os grupos; as freqüências atingiram valores em torno de $60 \%$. A retirada da semente do tomate, uma medida dietética simples, reduziria a possibilidade de perda de cálcio.

Entre as bebidas, o café é consumido pelo menos três vezes na semana por mais de $87 \%$ das mulheres com osteopenia e osteoporose. Lloyd et al. (1997) relatam não ter encontrado associação entre consumo de cafeína e mensuração óssea em estudo realizado com 138 mulheres na pós-menopausa com 55 a 70 anos. Segundo Weaver et al. (1999) o alto consumo de cafeína teria um efeito modesto sobre a perda de cálcio na maioria das pessoas. No entanto, Harris $\&$ Dawson-Hughes (1994), ao verificarem o efeito da ingestão da cafeína na alteração do grau da densidade mineral óssea em 205 mulheres saudáveis, não-fumantes e na pós-menopausa, consideraram que um grande segmento da população idosa pode estar consumindo uma combinação de baixa ingestão de cálcio (<800mg) com alta ingestão de cafeína (>450mg), o que seria prejudicial à massa óssea. A combinação entre ingestão de café e consumo de cálcio abaixo do recomendado está sendo reproduzida entre as mulheres entrevistadas. Embora haja controvérsia so bre os efeitos da cafeína na alteração da massa óssea, seu conteúdo em ácido oxálico não recomenda consumo excessivo. O chocolate, também rico em ácido oxálico, não é expressivamente consumido pelo grupo participante.

É importante frisar que um dos intuitos deste estudo foi verificar a presença ou não de inibidores da absorção de cálcio nas refeições, não sendo levantados os valores de consumo destes inibidores, até mesmo pela dificuldade em ter à disposição tabelas com seus teores. Este fator, entre outros, demonstra a dificuldade no planejamento das dietas para este grupo específico.

0 tratamento estatístico valeu-se de odds ratio (Tabela 5), o qual mede a mudança proporcional na incidência de um fato produzido

Tabela 4. Presença de fatores antinutricionais na dieta de mulheres na pós-menopausa.

\begin{tabular}{|c|c|c|c|c|}
\hline \multirow{3}{*}{ Alimentos limitantes* } & \multicolumn{4}{|c|}{ Diagnóstico } \\
\hline & \multicolumn{2}{|c|}{ Osteopenia $(n=43)$} & \multicolumn{2}{|c|}{ Osteoporose $(n=33)$} \\
\hline & $\mathrm{n}$ & $\%$ & $\mathrm{n}$ & $\%$ \\
\hline Café & 38 & 88,4 & 29 & 87,9 \\
\hline Tomate & 28 & 65,1 & 19 & 57,6 \\
\hline Pão integral & 16 & 37,2 & 10 & 30,3 \\
\hline Espinafre & 16 & 37,2 & 7 & 21,2 \\
\hline Refrigerante & 13 & 30,2 & 6 & 18,2 \\
\hline Chá & 11 & 25,6 & 8 & 24,2 \\
\hline Mate & 8 & 18,6 & 11 & 33,3 \\
\hline Biscoito de fibras & 5 & 11,6 & 3 & 9,1 \\
\hline Chocolate & 4 & 9,3 & 5 & 15,2 \\
\hline Arroz integral & 2 & 4,7 & 3 & 9,1 \\
\hline
\end{tabular}

${ }^{(*)}$ Freqüência de consumo em pelo menos 3 vezes por semana. 
pelo deslocamento de um nível de exposição a outro, representando a estimativa do risco relativo. Se uma variável é designada para o primeiro posto (presença da variável atributo fator de risco), seu valor preditivo é menor ou igual a 0,5 . A variável é designada para o segundo posto, se seu valor preditivo respectivo é maior do que 0,5 . 0 odds ratio é calculado como $\left(\mathrm{f}_{11} * \mathrm{f}_{22}\right) /\left(\mathrm{f}_{12} * \mathrm{f}_{21}\right)$, onde $\mathrm{f}_{\mathrm{ij}}$ apresenta-se para as freqüências respectivas na tabela dois por dois. Os odds ratios superiores a um indicam que a classificação é melhor do que uma esperada ao acaso. Entretanto, estas são classificações post-hoc, porque as enumerações foram computadas tão somente para maximizar a ocorrência da probabilidade do evento. Trata-se de um estudo de Epidemiologia Nutricional, onde se indaga sobre os fatores, dentre eles a dieta, aos quais atribuem-se os efeitos observados. A investigação epidemiológica é caracterizada como pesquisa observacional, onde o pesquisador não tem controle direto da variável independente, porque suas manifestações já ocorreram com anterio ridade (Almeida Filho \& Rouquayrol, 1992). A causa é interpretada como uma multiplicidade de fatores propícios que, reunidos em configurações determinadas, aumentam a probabilidade de ocorrência (risco) de determinado acontecimento. Assim, o núcleo duro da investigação será a proposição da associação causal entre os fatores de risco e os seus possíveis desfechos, no presente caso, a osteoporose.
A variável designada para o primeiro posto foi a não-reposição hormonal $(2,000)$, ou seja, as participantes que não fazem terapia de reposição hormonal têm um risco duas vezes maior de vir a ter osteoporose entre as com osteopenia. A não-exposição ao sol $(1,516)$ e o consumo de bebida alcoólica na juventude $(1,346)$ foram mais expressivos do que o consumo inadequado de cálcio na dieta (1,163). Seguiram-se a estes fatores a ausência de atividade física atualmente $(1,145)$, a história familiar de osteoporose $(1,101)$ a ausência de atividade física na juventude $(1,006)$, o tabagismo $(0,851)$ e o consumo de álcool atualmente $(0,827)$.

A terapia de reposição hormonal, como ação de intervenção no desfecho da osteoporose, é um assunto polêmico entre os pesquisadores. Sittisomwong et al. (2000), após revisão sobre o assunto, colocaram-se a favor deste tipo de intervenção, em pacientes previamente selecionadas, esclarecendo que os benefícios parecem estar acima do risco de câncer ou de sua recorrência. Outros autores (Torgerson, 2000; Lippman, 2001) apontam para o aumento do risco de contrair câncer de mama e de endométrio, mas não negam a proteção contra o risco de fraturas na pós-menopausa e de doenças cardiovasculares. Burkman (2001) e Pritchard (2001) revelam que estudos mais recentes indicam redução do risco de câncer coloretal e de doença de Alzheimer. Hodiernamente, estão sendo propostas investigações para verificar os efeitos da terapia de reposição hormonal versus nenhuma

Tabela 5. Fatores concorrentes à osteoporose em mulheres na pós-menopausa.

\begin{tabular}{lcc}
\hline Fatores de risco & Odds Ratio & Intervalo de Confiança (95\%) \\
\hline Não reposição hormonal & 2,000 & {$[0,770 ; 5,193]$} \\
Não exposição ao sol & 1,516 & {$[0,609 ; 3,773]$} \\
Consumo de bebida alcoólica na juventude & 1,346 & {$[0,523 ; 3,467]$} \\
Consumo inadequado de cálcio considerando apenas os alimentos lácteos & 1,163 & {$[0,183 ; 7,391]$} \\
Ausência de prática de atividade física atualmente & 1,145 & {$[0,461 ; 2,844]$} \\
História familiar de osteoporose & 1,101 & {$[0,305 ; 3,978]$} \\
Ausência de atividade física na juventude & 1,006 & {$[0,398 ; 2,545]$} \\
Tabagismo & 0,851 & {$[0,219 ; 3,299]$} \\
Consumo de bebida alcoólica atualmente & 0,827 & {$[0,293 ; 2,335]$} \\
\hline
\end{tabular}


terapia hormonal, ambas vinculadas a outras estratégias como suplementos de cálcio, adequação da dieta e exercícios. Espera-se esclarecer melhor a questão, quando investigadas de forma interativa.

Se for considerado o balanço entre facilitadores e inibidores da absorção do cálcio dietético, mais difícil se torna o tratamento da osteoporose unicamente pela dieta, sem o uso de suplementos. Neste estudo, a não-reposição hormonal foi o fator de risco com maior freqüência, seguida da não-exposição ao sol, permitindo inferir sobre o medo do risco aumentado de contrair câncer e a opção de viver mais recolhido ao lar, em decorrência da redução do poder aquisitivo inerente aos idosos. No entanto, a maioria dos fatores de risco, à exceção de três (consumo de álcool e ausência de atividade física, ambos na juventude, e história familiar de osteoporose), são plenamente modificáveis. Esta premissa permite acreditar que o sucesso na prevenção da osteoporose está na adoção de novos hábitos de vida, buscando a interação entre terapia de reposição hormonal, dieta e exercício ao ar livre.

\section{O N C L U S Ã O}

Os avanços dos estudos sobre a interação entre nutrientes reforçam a dificuldade de se obter sucesso na prevenção da osteoporose sem uma intervenção multifaceada e precoce. 0 consumo de dietas que atendam as recomendações de cálcio e nos quais este nutriente esteja biodisponível deve ser incentivado como uma das estratégias da prevenção de osteoporose primária, tornando-se necessário investir em educação nutricional para crianças, jovens e adultos.

A contribuição desta pesquisa está em indicar os principais fatores de risco para desenvolver osteoporose primária em mulheres na pós-menopausa diagnosticadas com osteopenia. A descrição da hierarquização destes dados, possibilitada pelo odds ratio, aponta a reposição hormonal como o fator de risco de maior proporção entre os elencados. Sendo um estudo observacional de corte e portanto pontual, não se podem verificar os efeitos da terapia de reposição hormonal ao longo do tempo, mas os resultados acerca da participação destes determinantes de risco permitem orientação a grupos de risco, propiciando uma visão holística do desfecho. A prevenção da osteoropose requer um conjunto de conhecimentos interativos, onde não se pode privilegiar um fator em detrimento de outros.

\section{RE FERÊ N C I A S B I B LIO G RÁ F I C A S}

ALMEIDA FILHO, N. ROUQUAYROL, M.Z. Introdução à Epidemiologia Moderna. 2.ed. Rio de Janeiro : Abrasco, 1992. p.104-115.

AMAYA-FARFAN, J. Fatores nutricionais que influem na formação e manutenção do osso. Revista de Nutrição Puccamp, Campinas, v.7, n.2, p.148-172, 1994.

AVENELL, A., RICHM OND, P.R., LEAN, M .E.J., REID, D.M . Boneloss associated with a high fibre weight reduction diet in postmenopausal women. European Journal of Clinical Nutrition, Bethesda, v.48, p.561-566, 1994.

BORGES, M .R., DI PIETRO, P. F., M ORETTO, E. Avaliação da ingesta alimentar de leite e cálcio de mulheres durante o climatério como fator de risco nutricional para a osteoporose na pós-menopausa. In: SIMPÓSIO SUL BRASILEIRO DE ALIMENTAÇÃO E NUTRIÇÃO: HISTÓRIA, CIÊNCIA E ARTE, 1., 2000, Florianópolis. Anais... Florianópolis : UFSC, 2000. p.591-594.

BURKM AN, R.T., COLLINS, J.A, GREENE, R.A. Current perspectives on benefits and risks of hormone replacement therapy. American Journal of Obstetrics Gynecology, v.185, 2 Suppl, p.S13-23, 2001.[online]. Available from: $<w w w$ :bireme.br/cgibin/wxislind.exe/iah/online>. Acess in 18 Nov 2001.

COELHO, R.G. Interações nutricionais. Revista de M etabolismo e Nutrição, Porto Alegre, v.2, n.3, p.106-117,1995. 
COMISIÓN EUROPEA. Informe sobre la osteoporosis en la Comunidad Europea: Accion para la prevención. Luxemburgo : Oficina de Publicationes Oficiales de las Comunidades Europeas, 1998. $116 p$.

DAWSON-HUGHES, B. Osteoporosis treatment and the calcium requirement. American Journal of Clinical Nutrition, Bethesda, v.67, p.5-6, 1998.

DRINKWATER, B.L., GRIMSTON, S., CULLEN, D.M.R., SNOW-HARTER, C.M. Osteoporosis and Exercise. Medical Science Sports Exercise, v.27, n.4, p.i-vii, 1995.

FRANCO, G. Tabela de composição química dos alimentos. 9.d. São Paulo : Atheneu, 1998. 194p.

INSTITUTO BRASILEIRO DE GEOGRAFIA E ESTATÍSTICA. Tabelas de composição de alimentos do IBGE. 4.ed. Rio de Janeiro, 1996. 137p.

HAACK, V.S., CHESTERS, J.G., VOLLENDORF, W., STORY, J.A., MARLETT, J.A. Increasing amounts of dietary fiber provided by foods normalizes physiologic response of the large bowel without altering calcium balance or fecal steroid excretion. American Journal of Clinical Nutrition, Bethesda, v.68, n.3, p.615-622, 1998.

HAINES, C.J, CHUNG, T.K.H, LEUNG, P.C., LEUNG, D.H.Y.,WONG,M Y., LAM , L.L. Dietary calcium intakes in postmenopausal Chinese women. European Journal of Clinical Nutrition, London, v.48, p.591-594, 1994.

HALLBERG, L., ROSSANDER-HULTÉN, L., BRUNE M., GLEERUP, A. Calcium and iron absorption: mechanism of action and nutritional importance. European Journal of Clinical Nutrition, London, v.46, p.317-327, 1992 .

HARRIS, S.S., DAWSON-HUGHES, B. Caffeine and bone loss in healthy postmenopausal women. American Journal of Clinical Nutrition, Bethesda, v.60, n.4, p.573-578, 1994.

HEATH, A-LM, SKEAFF, C.M ., GIBSON, R.S. The relative validity of a computerized food frequency questionnaire for estimating intake of dietary iron and its absorption modifiers. European Journal of Clinical Nutrition, London, v.54, p.592-599, 2000.
INSTITUTO NACIONAL DO CANCER [online] Tabagismo. Disponível em: www: <http:// www.falandoseriosobredrogas.org. br/tab7.htm>. Acesso em: 4/11/2001.

KANIS, J. A.,M ELTON, L. J.,CHRISTIANSEN,C., JOHNSTON,C. C., KHALTAEV, N. The diagnostic of osteoporosis. Journal of Bone and Mineral Research, New York, v.9, n.8, p.1137-1141, 1994.

KÄRLKÄINEN M., LAMBERG-ALLARDT C. An acute intake of phosphate increases parathyroid hormone secretion and inhibits bone formation in young women. Journal of Bone and Mineral Research, New York, v.11, p.1905-1912, 1996.

KHOURY, M.J. Genetic and Epidemiological Approaches to the Search for Gene-Environment Interaction: the Case of Osteoporosis. American Journal of Epidemiology, Baltimore, v.147, p.1-2, 1998.

KUNG, A.W.C., LUK, K.D.K., CHU, L.W., CHIU, P.K.Y. Age-related osteoporosis in Chinese: an evaluation of the response of intestinal calcium absorption and calcitropic hormones to dietary calcium deprivation. American Journal of Clinical Nutrition, Bethesda, v.68, p.1291-1297,1998.

LEM ON, P.W.R. Effects of Exercise on Dietary Protein Requirements. International Journal of Sport Nutrition, n.8, p.426-447, 1998.

LIPPMAN, M.E., KRUEGER, K.A, ECKERT, S., SASHEGYI, A.A. Indicators of lifetime estrogen expose: effect on breast cancer incidence and interaction with raloxifene therapy in the multiple outcomes of raloxifene evaluation study partipants. J Clin Oncol, v.19, n.12, p.3111-3116, 2001. [online]. Available from:< www.bireme.br/cgi-bin/wxislind.exe/iah/ online>. Acess in 18 Nov 2001.

LITVAK, J. El envejecimiento de la población: un desafío que va más allá del año 2000. Boletín de la Oficina Sanitaria Panamericana, Ginebra, v.109, n.1, p.1-5, 1990. (Informe especial).

LLOYD,T., ROLLINGS, N., EGGLI, D.F., KIESELHORST, K., CHINCHILLI, V.M. Dietary caffeine intake and bone status of postmenopausal women. American Journal of Clinical Nutrition, Bethesda, v.65, p.1826-1830,1997. 
LUCAS, B. Nutrição na infância. In: MAHAN, L.K., ESCOTT-STUMP, S. Krause: alimentos, nutrição e dietoterapia. 9.ed. São Paulo : Roca, 1998. p. 259-278.

LUCASIN Junior, R., LIMA, W.L. Osteoporose: exercício como prevenção e tratamento. Arscurandi: A Revista da Clínica Médica, p.28-36, 1994.

MARQUES NETO, J.F, LEDERMAN, R. Osteoporose Brasil: ano 2000. São Paulo : Limay, 1995. 137p.

NIEVES, J.W., KOMAR, L., COSM AN, F., LINDSAY, R. Calcion potentates the effect of estrogen and calcitonin on bone mass: review and analysis. American Journal of Clinical Nutrition, Bethesda, v.67, p.18-24, 1998.

PHILIPPI, S.T., LATTERZA, A.R., CRUZ, A.T.R., RIBEIRO, L.C. Pirâmide alimentar adaptada: guia para escolha do alimentos. Revista de Nutrição, Campinas, v.12, n.1, p.65-80,1999.

PINHEIRO, A.B.V., LACERDA, E.M .A., BENZECRY, E.H., GOMES, M.C., COSTA, V.M. Tabela para avaliação de consumo alimentar em medidas caseiras. 2.ed. Rio de Janeiro : Elisa Lacerda, 1994. 63p.

PRINCE, R., DEVINE, A., DICK, I., CRIDDLE, A., KERR, D., KENT, N., PRICE, R., RANDELL, A. The Effects of Calcium Supplementation (M ilk Powder or Tablets) and Exercise on Bone Density in postmenopausal women. Journal of Bone and Mineral Research, New York, v.10, n.7, p.1068-1075, 1995.

PRITCHARD, K.I. Hormone replacement in women with a history of breast cancer. Oncologist, v.6, n.4, p.353-362, 2001. [online]. Available from: $<$ w w w . query.fcgi ? $\mathrm{cmd}=$ Retrieve $\& d b=P u b$ M ed\&list_uids $=11524554 \& d o p t=A b s t r a c>$. Acess in 18 Nov 2001.

SHAW, J.M., WITZKE, K.A. Exercise for Skeletal Health and Osteoporosis Prevention. In: ACSM'S RESOURCE. Manual for guidelines for exercise testing and prescription. 3.ed. Baltimore : Willians and Wilkins, 1998. p.288-239.
SILVA, M.R., SILVA, M .A.A.P. Aspectos nutricionais de fitatos e taninos. Revista de Nutrição, Campinas, v.12, n.1, p.21-32, 1999.

SITTISOM WONG, T., SUNEJA, A., KUDELKA, A.P., VERSCHRAEGEN, C.F. Estrogen replacement therapy and ovarian cancer. Eur J Gynaecol, v.21, n.4, p.348-354, 2000. [online]. Available from: <www.bireme.br/cgi-bin/wxislind.exe/iah/online>. Acess in 18 Nov 2001.

TORGERSON, D.J. HRT and its impact on the menopause, osteoporosis and breast cancer. Expert Opin Pharmacother, v.15, n.6, p.1163-1169, 2000. [online]. Available from: <www.bireme.br/cgi-bin/ wxislind.exe/iah/online>. Acess in 18 Nov 2001.

ULRICH, C.M ., GEORGIO, C.C., SNOW-HARTER, C.M., GILLIS, D.E. Bone mineral density in mother-daughter pairs: Relations to lifetime exercise, lifetime milk consumption, and calcium supplements. American Journal of Clinical Nutrition, Bethesda, v.63, p.72-79, 1996.

WEAVER, C.M., PROULX, W.R., HEANEY, R. Choices for achieving adequate dietary calcium with a vegetarian diet. American Journal of Clinical Nutrition, Bethesda, v.70, p.543S-548S, 1999. Supplement.

WHITING, S.J. The inhibitory effect of dietary calcium on iron bioavailability: a cause for concern? Nutrition Reviews, Boston, v.53, n.3, p.77-80, 1999.

WILLET, W. Nutritional epidemiology. Oxford : Oxford University Press, 1990. 396p.

ZMUDA, J.M., CAULEY J.A., FERRELL, R.E. Vitamin D receptor Gene Variants and Osteoporosis. Epidemiologic Reviews, Baltimore, v.22, n.2, p.203-217, 2000.

Recebido para publicação em 17 de julho de 2001 e aceito em 9 de abril de 2002. 